\title{
Experimental Measurement of a UAV Propeller's Thrust
}

\author{
Patrik KÓSA*, Marián KIŠEV, Lukáš VACHO, Ladislav TÓTH, Martin OLEJÁR, Marta HARNIČÁROVÁ, Jan VALíČEK, Hakan TOZAN
}

\begin{abstract}
At present, there are several types of propellers in the field of the use of Unmanned Aerial Vehicles (UAVs) with unknown parameters, where it is necessary to provide information about their thrust, current consumption and maximal rotational speed (RPM). Commonly used methods for measurement of a propeller's thrust are mostly based on the usage of a single purpose system, on short measurements without data storage or on inaccurate sensors. The goal of this article is to develop a universal experimental measuring system for more accurate measurement of propeller's parameters (thrust, current consumption, maximal RPM). For more accurate measurement, the battery voltage, temperature and humidity of the environment were also measured. To acquire, measure and store the data safely on a micro SD card, a processing circuit based on an ATmega2560 microcontroller was developed. This innovative approach allowed to analyse the behaviour of the propeller and to measure the dependencies of the RPM on pulse width, of the current on RPM and of the thrust on RPM at different input conditions. The measurements have shown that the dependencies can be approximated by cubic functions. The mathematical description allows predicting the behaviour of the propeller in unmeasurable conditions.
\end{abstract}

Keywords: BLDC motor; propeller; thrust; UAV

\section{INTRODUCTION}

The increasing use of multirotor UAVs (drones) in different applications requires detailed information about the parameters of several motor-propeller configurations. These parameters are usually the thrust of the propeller, the maximal RPM and the maximal electrical current required by the used brushless motor. Generally, the manufacturers of brushless direct current (BLDC) motors provide information about their performance and in some cases also data of thrust measured with pre-selected propellers. Usually the information about the used propeller is provided by its diameter and pitch. Since the information about the combination of various motors and propellers is in the most cases not included in the product description, it is necessary to obtain this data with experimental measurements.

Nowadays, there exist many methodologies for the measurement of the parameters of the propellers. Jakubowski [1] in his paper presents a test stand for measurement of a propeller's thrust. His solution was based on a simple frame, where only the thrust is measured by a force transducer that was connected to the motor by steel cable. In his solution, the data was acquired by software Matlab-Simulink and the motor was driven by an application that generates pulse width modulation (PWM) signal for the Electronic Speed Controller (ESC). Zabunov [2] describes an instrument that is based on a simple two arm balancing mechanism that is suitable for thrust measurement of propellers for micro-UAVs. The thrust was measured by a common scale. Unlike the previous solution, the author has not used any application to process or store the data from the measurements. Theys et al. [3] introduce a more complex research done by a thrust measurement system based on the same kinematics as previous authors. In their paper, they are investigating the interaction between two arms on a drone, the behaviour of two and three blade propellers against each other and their interaction against the environment. The measurement of the thrust was based on a common force cell that was mounted to the end of the pivot. Unfortunately, the authors do not describe the way of processing data and its acquisition. Different construction of a system for measurement of the propeller's parameters is described in [4]. The author introduces a propeller dynamometer that is useful for propellers with diameter up to $250 \mathrm{~mm}$ and it is measuring the generated thrust and torque by several load cells. The acquired data is then sent via Bluetooth to the computer. Other methods how the parameters of propellers are measured are described in [59].

According to the described results, it is obvious that in most cases these systems are designed as one purpose devices to measure the parameter of only one specific category of motor or propeller. The acquisition and storage of the measured data is also a big issue, because it is mostly done by manual measurements that require presence of an operator. These facts led us to set a goal to design a measurement stand that will be able to solve the issues with problematic measurements and storage of the required parameters of several types of BLDC motors and propellers.

This article describes the design of an innovative approach to the measurement of the parameters of a propeller without documentation. The designed solution that is based on calibrated sensors allows us to measure the required data and to create mathematical equations to describe the behaviour of the propellers at different input conditions.

Finally, the designed device allowed us to obtain the missing propeller's parameters and to analyse its behaviour. The mathematical equations derived by regression from the created dependencies showed that the measured dependencies can be described by a cubic regression model. This equation allows us to predict the propellers behaviour at unmeasurable conditions.

\section{MATERIALS AND METHODS}

The goal of this article was to develop a measurement system that will be suitable to measure the parameters of several BLDC motor-propeller combinations that are used on multirotor drones. The measured data will be used to derive the mathematical equations that will describe the propellers behaviour in different conditions. These parameters are usually the thrust of the propeller, maximal 
RPM and the maximal electrical current required by the used brushless motor. The used sensors and methods about how the designed measurement chain was prepared will be described in the next sections.

\subsection{Experimental Setup of the Measurement}

For the experimental setup we have used a Tarot 5008 $340 \mathrm{KV}$ BLDC motor with a Dragonfly 1755 propeller. According to the manufacturer information, the motor requires a six cell Li-Pol battery and a minimum 40 A ESC. As a voltage supply, we have used a Gens Ace TATTU $12000 \mathrm{mAh}$ Li-Pol battery and a MANSON HCS-3602 1$32 \mathrm{~V}, 0-30$ A regulated power supply. To control the BLDC motor, we have used a 50 A EMAX BLHeli ESC. The thrust of the propeller will be measured by a calibrated 20 $\mathrm{kg}$ force gauge FG-6020SD with $\pm 0,5 \%+2$ digit accuracy within $23,5{ }^{\circ} \mathrm{C} \pm 5{ }^{\circ} \mathrm{C}$, which sends the measured data via RS232 communication bus (hereinafter referred to as RS 232). The influence of the designed system on the measured force will be analysed by a set of calibrated weights with $0,015 \%$ accuracy. The RPM of the BLDC motor will be measured by a reflective optical sensor Pepperl+Fuchs GLV12-8-200 with $\pm 0,02 \%$ accuracy, from which the data is processed by an Almemo 2590 instrument and sent also by RS232 bus. Another important quantity is the value of electrical current that is consumed by the motor. It is possible to determine the capacity of the needed Li-Pol battery from the value of the electrical current consumption. For the measurement itself, it was necessary to use a current sensor that will be suitable to measure wide range of electrical current. For this reason, we have used an ACS 770 hall-effect current sensor with $\pm 0,5 \%$ output error that is suitable to measure direct currents (DC currents) up to $100 \mathrm{~A}$. To know how the propeller behaves in every situation, it was also important to measure the voltage of the used Li-Pol battery by operational amplifiers, at least the temperature and humidity of the environment have to be measured. That was done by a SHT 20 sensor with $\pm 3 \%$ tolerance for relative humidity and $\pm 0,3 \%$ tolerance for temperature. The measured data from the sensors is processed by an Arduino Mega 2560 R3 (hereinafter referred to as Arduino) and saved to a micro SD card. This custom sensor setup will allow to measure automatically without the need of the presence of an operator. Since the Arduino works with 5 V (TTL) logic, all signals need to be adjusted to TTL logic levels.

\subsection{Aerodynamic Aspects of the Measurement}

When a propeller is rotating in an environment, aerodynamic interactions can appear when it is operating near the ground or ceiling. These interactions are called the ground effect and the ceiling effect [10].

The ground effect can be experienced when a vertical take-off aircraft like a helicopter or a UAV is taking off from the ground. Near the ground, the downward flow is slowed and forced to radially spread out. This phenomenon causes a raise of the lift force when taking off. The streamlines near the ground are turning around and changing their vertical direction from the propeller to parallel (horizontal) direction with the ground. This orientation change causes an increase of the flow velocity at the exit area [11]. The increased velocity then translates to increased thrust of the rotating propeller. The ground effect of a UAV's propeller is usually analysed through corrected model based on Cheeseman [12]. The model corrected by Danjun [11] takes the following form:

$$
\frac{F}{F_{0}}=\frac{1}{1-\rho\left(\frac{R}{4 Z}\right)^{2}}
$$

where $F$ is the thrust at the ground effect region, $F_{0}$ is the thrust outside the ground effect region, $\rho$ is the correction coefficient for quadrotor UAV's, $R$ is the radius of the propeller and $Z$ is the distance of the rotor above the ground.

The Cheeseman's model states that the ground effect is significant to $Z / R=1,5$. Further study that is described in [13] shows that the ground effect is in case of UAVs still measurable when $Z / R=4$.

The ceiling effect is the opposite of the ground effect and appears when a rotorcraft flies close to a ceiling or something similar that is above the propellers. The presence of a ceiling causes the propellers to pull the aircraft to the surface where the thrust and the RPM are also increased. Researches in [10, 14, 15] show that the influence of the ceiling effect becomes less significant when $-Z / R \geq 1$.

\section{RESULTS}

\subsection{Experimental Setup of the Measurement}

For the purposes of this research, it was necessary to develop an innovative custom measurement stand that will be equipped with the used sensors and will be useful for several types of motor-propeller combinations. The designed custom measurement stand is shown in Fig. 1.

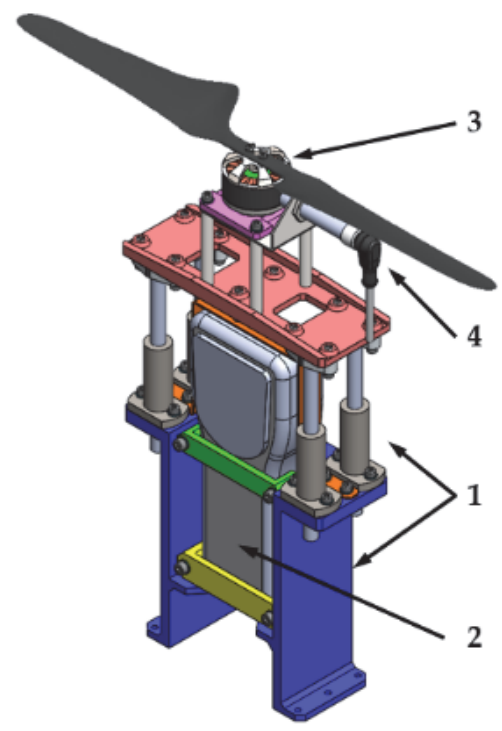

Figure 1 Experimental setup of the measurement stand: Isometric view (1 - measurement stand body, 2 - force sensor, 3 - BLDC motor with propeller, 4 - RPM sensor)

The measurement stand consists of a 3D printed body made of PETG (Poly-Ethylene Terephthalate Glycol) to which the sensors are attached. The force sensor is attached 
to the body by three fixtures to hold the sensor in place when the vertical force is applied. To measure only the vertical thrust, the motor and the propeller were placed on a plate guided by four LMH10LUU linear plain bearings. The optical RPM sensor was placed directly on the side of the motor on a sheet metal holder plate to measure its RPM. Since we use an out-runner motor (the rotor of the motor is located outside), we had to place a reflective point on it to measure its RPM.

To prevent the measurement from the most significant influence, the ground effect, the propeller was mounted upside down. At this position, the propeller has to rotate counter clockwise and the generated thrust will vertically push the force sensor (the airflow streamlines will have upward direction). When we consider that the remaining ceiling effect is calculated with a surface that fully covers the propellers radius, we can reduce it by minimizing the guide plate to $185 \times 65 \mathrm{~mm}$. Additional reduction of the ceiling effect was done by placing the motor on four $60 \mathrm{~mm}$ distance bolts to raise the distance of the propeller against the guide plate to $100 \mathrm{~mm}$. When we calculate the $\mathrm{Z} / \mathrm{R}$ constant from the defined dimensions (propeller radius is $215,9 \mathrm{~mm}$ and its distance is $100 \mathrm{~mm}$ from the guide plate) we get a 0,463 ratio. This constant represents a fully covered zone under the propeller's diameter. When we compare the surface of the guide plate $\left(0,012 \mathrm{~m}^{2}\right)$ to the surface of the rotating region $\left(0,146 \mathrm{~m}^{2}\right)$, the result is that the guide plate surface covers only $8,22 \%$ of the propeller's region. To additionally reduce the ceiling effect, we have placed the measurement stand on a 1,035 $\mathrm{m}$ high support frame. The backbone of the frame is a 1 meter long $60 \times$ $60 \mathrm{~mm}$ Aluminium extrusion profile. On the top of this profile, a top holder plate is mounted on which the measurement stand is fixed by 6 screws. The bottom of the frame was made of a $10 \mathrm{~mm}$ thick stainless-steel plate to make the frame stable. The experimental assembly of the support frame and the measurement stand is shown in Fig. 2.
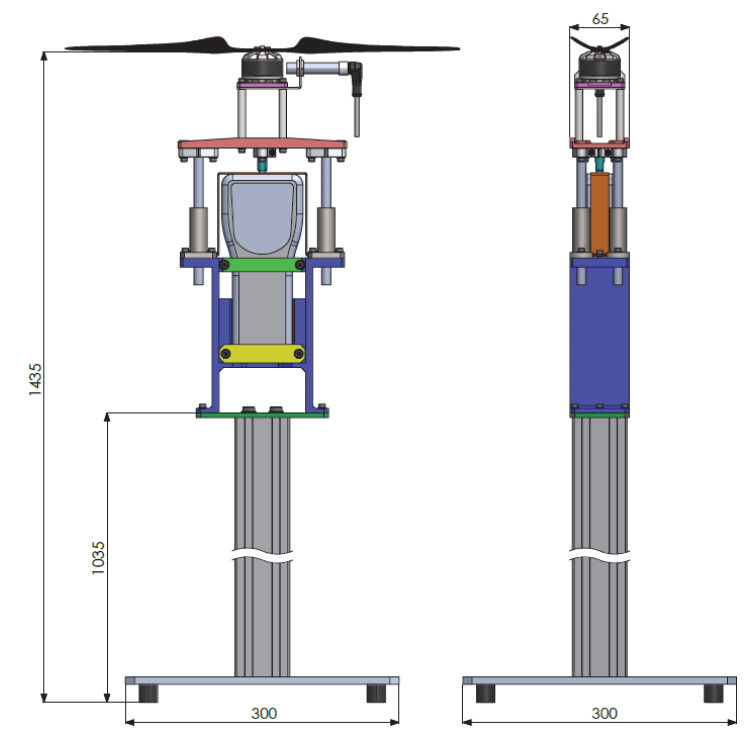

Figure 2 The experimental assembly of the support frame and the measurement stand

The compatibility with other motor/propeller configurations was achieved by the possibility to mount several types of motors on one holder plate. Usually, the most frequently used BLDC motors have four M3 thread holes in their bottom placed with a spacing of $16 \times 16$, $16 \times 19,19 \times 19,19 \times 25,25 \times 25$ or $32 \times 32 \mathrm{~mm}$. Therefore, we have designed a universal mounting plate for the BLDC motor (Fig. 3) that is compatible with the named patterns.

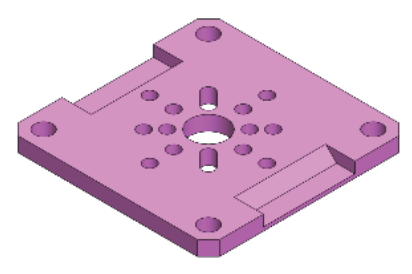

(a)

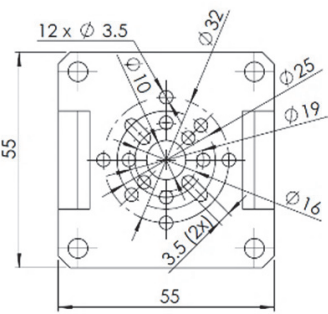

(b)
Figure 3 Designed universal mounting plate: (a) Isometric view; (b) Simplified drawing

To ensure that the friction in the linear plain bearings would not influence the measurement of the propeller's thrust, we have done several measurements with calibrated weights. The used calibrated weights had mass of $100 \mathrm{~g}$, $200 \mathrm{~g}, 500 \mathrm{~g}, 1 \mathrm{~kg}, 2 \mathrm{~kg}$ and $5 \mathrm{~kg}$. For each weight we have done 10 measurements to analyse the average measured value of their weight (see Tab. 1).

Table 1 Measurement of the calibrated weights

\begin{tabular}{|c|c|c|c|}
\hline $\begin{array}{c}\text { Type of } \\
\text { weight }\end{array}$ & $\begin{array}{c}\text { Average measured } \\
\text { weight } m / \mathrm{g}\end{array}$ & $\begin{array}{c}\text { Difference } \\
\Delta m / \mathrm{g}\end{array}$ & $\begin{array}{c}\text { Percentage difference } \\
\Delta m_{\mathrm{p}} / \%\end{array}$ \\
\hline $100 \mathrm{~g}$ & 99,142 & 0,858 & 0,858 \\
\hline $200 \mathrm{~g}$ & 198,457 & 1,543 & 0,772 \\
\hline $285,7 \mathrm{~g}$ & 498,149 & 1,851 & 0,370 \\
\hline $322,7 \mathrm{~g}$ & 998,764 & 1,236 & 0,124 \\
\hline $358,4 \mathrm{~g}$ & 1999,153 & 0,847 & 0,042 \\
\hline $393,1 \mathrm{~g}$ & 4999,514 & 0,486 & 0,010 \\
\hline
\end{tabular}

According to the measured values in Tab. 1, we can see that between the nominal mass of the weight and the measured value is a slight difference caused by the bearings. Since the percentage difference is lower than the accuracy of the used sensor, this difference is negligible for the measurement of the propeller's thrust.

\subsection{Acquisition and Storage of the Measured Data}

To acquire and store the measured data, it was necessary to design a custom circuit that will process the measured data from the sensors to the Arduino Mega 2560 R3 development board. The block diagram of the designed circuit for data processing is shown in Fig. 4.

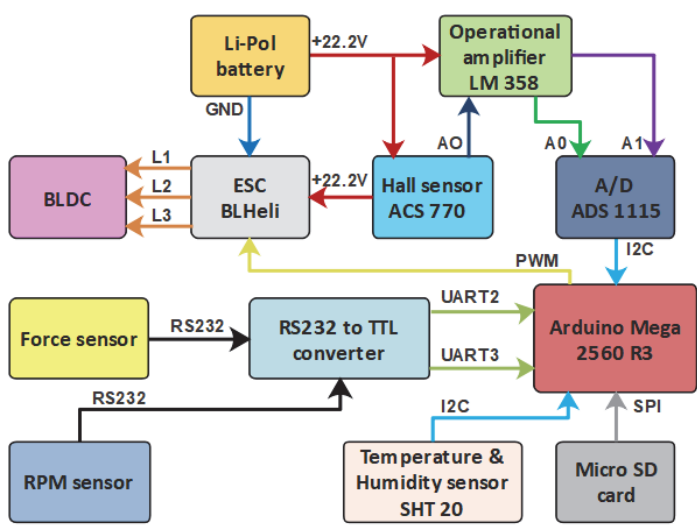

Figure 4 Block diagram of the circuit for data processing 
The main part of the data processing circuit is the Arduino development board, which receives the measured data from the sensors via UART, I2C and SPI communication buses. The data of the force and RPM sensor is by a MAX232 integrated circuit converted from RS232 to UART standard, which is supported by the Arduino and sent to pins UART 2 and UART 3. To measure the current consumption of the BLDC motor a 100 A Hall-effect current sensor ACS 770 was used. It has a sensitivity of $20 \mathrm{mV} / \mathrm{A}$ and a zero current offset voltage of 2,5 V (half of the supply voltage). This voltage signal had to be processed by an $\mathrm{A} / \mathrm{D}$ (analogue to digital) converter. Since the voltage change is according to the built in 10-bit A/D converter low (approx. $4,8876 \mathrm{mV}$ resolution) we had to use an external 16-bit 4 channel converter ADS 1115 with much higher resolution. This A/D converter contains a programmable gate amplifier which allows adjusting the input range from $\pm 0,256 \mathrm{~V}$ to $\pm 6,144 \mathrm{~V}$. We chose $\pm 1,024 \mathrm{~V}$ input voltage range, which resulted in a resolution of approx. 15,625 $\mu \mathrm{V}$. For this voltage range, the output voltage of the current sensor had to be adjusted by operational amplifiers. The simplified schematic diagram of the voltage shifting circuit is shown in Fig. 5.

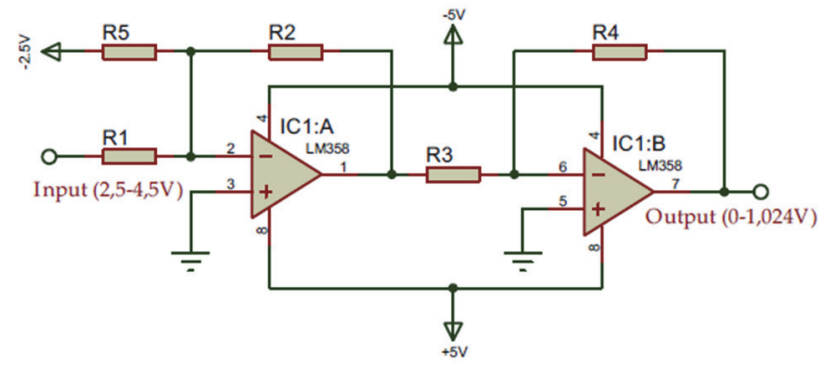

Figure 5 Simplified schematic diagram of the voltage shifting circuit

The adjustment of the voltage was performed by a LM358 operational amplifier, which was used as an inverting amplifier in two serially connected blocks. The voltage offset shifting at zero current was achieved by an additional input branch in the summing amplifier circuit. Fig. 5 shows that to achieve the correct voltage range, it is necessary to provide a power supply of $\pm 5 \mathrm{~V}$ and a voltage of $-2,5 \mathrm{~V}$ to the input of the summing amplifier. The voltage of the battery was also processed by two serially connected inverting amplifiers, but in this case the input voltage of the battery had to be lowered by a voltage divider to maximally $10 \mathrm{~V}$. For safety purposes, the maximal measured voltage was set to $26 \mathrm{~V}$. For this reason, the positive power supply had to be $+12 \mathrm{~V}$. In this case, the output voltage is also in $0-1,024 \mathrm{~V}$ range for the better resolution of the $\mathrm{A} / \mathrm{D}$ converter. The measured voltage of both outputs is then sent to the Arduino by I2C standard. The possibility to measure currents up to $100 \mathrm{~A}$ and voltages up to $26 \mathrm{~V}$ allows to use the designed circuit with different BLDC motors and power supplies (Li-Pol batteries). The temperature and humidity sensor SHT20 was connected according to the recommended schematic from its datasheet. The only issue was that it uses 3,3 V logic not $5 \mathrm{~V}$ like the Arduino. For this reason, we have used a simple bi-directional logic level converter that consists of two N-channel MOSFET transistors BSS138.
To control the rotational speed of the motor, it was necessary to connect the ESC to a PWM pin. The frequency of the signal was set to $50 \mathrm{~Hz}$ and the pulse width had to be between $1 \mathrm{~ms}$ and $2 \mathrm{~ms}$, where $1 \mathrm{~ms}$ means zero RPM and 2 ms means maximal RPM of the motor.

Since the circuit is not directly integrated with the microcontroller, it is possible to use it with several kinds of embedded boards (Raspberry Pi, STM32 etc.). The created circuit board together with the Arduino was placed in a box with dimensions $120 \times 65 \times 65 \mathrm{~mm}(L \times W \times H)$ that will be located on the bottom plate of the support frame, so it would not represent an additional obstacle for the air flow.

\subsection{Development of the Measurement Algorithm}

To measure the parameters accurately, we had to develop a custom program for the Arduino. The program begins with the basic setup of input/output ports and setup of the PWM signal to $1 \mathrm{~ms}$. The reason why the PWM had to be set up at this moment is that the driver of the BLDC motor has to be initialized in a steady "zero RPM" state. The program is divided into two basic modes (manual and automatic mode) by the received data from the serial link. If the received data is " 0 " then the pulse width is set to 1 $\mathrm{ms}$ and the motor is stopped.

In the manual mode, the pulse width of the PWM signal is set up according to the numeric data received on the serial link, which need be to between 1000 to 2000 (pulse width in $\mu \mathrm{s}$ ). To acquire the most accurate data, we had to do several measurements to get an average value of them. We decided to measure 10 samples of all quantities. The first value that had to be measured is the RPM of the propeller, because the RPM sensors frequency of sending data is $1 \mathrm{~Hz}$. After that the data from the force sensor is retrieved, because it is sending data every $20 \mathrm{~ms}$. The other quantities like battery voltage, current, temperature and relative humidity are retrieved every $10 \mu \mathrm{s}$, because the sensors and the $\mathrm{A} / \mathrm{D}$ converter are communicating with 100 $\mathrm{kHz}$ frequency. The measured values are then going to be saved into a text file. Each measurement is in a separate line in the following format: RPM; thrust; temperature; relative humidity; battery voltage; current. This format helps to easily process the data after the measurement is done. The measurement of 10 samples was accomplished by a loop with pre-defined number of repeats. After the 10 measurements were finished, the measured data is saved to the microSD card, the pulse width is set to $1000 \mu$ s and the program jumps back to the part where the data from serial link is checked.

In the automatic mode, the pulse width of the PWM signal is automatically controlled by the program. The program runs in a loop, where the pulse width is incremented by a $50 \mu$ s step until it is lower than $2000 \mu$ s. In each pulse width, the program is also measuring 10 samples of the parameters and saves them on the microSD card as it was in the manual mode. After the $2000 \mu$ s pulse width was reached and the 10 measurements were done, the program sets the pulse width to $1000 \mu$ s and the program ends.

This two-mode program setup allows to do not only fixed complete measurements, but also to do a few test measurements to predict possible issues with the motorpropeller configurations. 


\subsection{Setup of the Measurement}

To be able to describe the propeller's characteristics completely, we had to reach the maximal possible values of each parameter. To reach them and to do stable measurements, it is not advised to use the Li-Pol battery, because it is discharging while the measurement is done and we will not be able to describe the value of each parameter that can be reached at exact conditions. For that reason, we had to measure the maximal current that is required by the BLDC motor with a fully charged Li-Pol battery $(25,2 \mathrm{~V}$ voltage). This was acquired by one test measurement at the manual mode with maximal possible pulse width $(2000 \mu \mathrm{s})$. The result of this measurement was the maximal current consumption of $26,13 \mathrm{~A}$. This value showed us that we can replace the Li-Pol battery with a regulated power supply with 30 A maximal current to be able to do the measurements at fixed voltage levels. Since the absolute minimal voltage of the battery is $22 \mathrm{~V}$ and the absolute maximal voltage is $25,2 \mathrm{~V}$, we decided to make measurements within this interval with $0,5 \mathrm{~V}$ steps (the final interval was from 22 to $25 \mathrm{~V}$ ). For each voltage we have done a full measurement at the automatic mode. The data of the 7 measurements will make it possible to understand the propeller's behaviour when the battery is discharging.

\subsection{Measurement of the Propeller's Characteristics}

To identify the most important parameters (maximal RPM, current consumption, generated thrust), we analysed the dependency of average RPM on pulse width, the dependency of average current consumption on RPM and the dependency of average generated thrust on RPM. The temperature of the air measured near the force sensor was $22,5^{\circ} \mathrm{C}$ and the relative humidity was $37 \%$. The values of temperature and humidity were calculated as an average of the measured values from each measurement. The dependency of average RPM on pulse width is shown in Fig. 6.

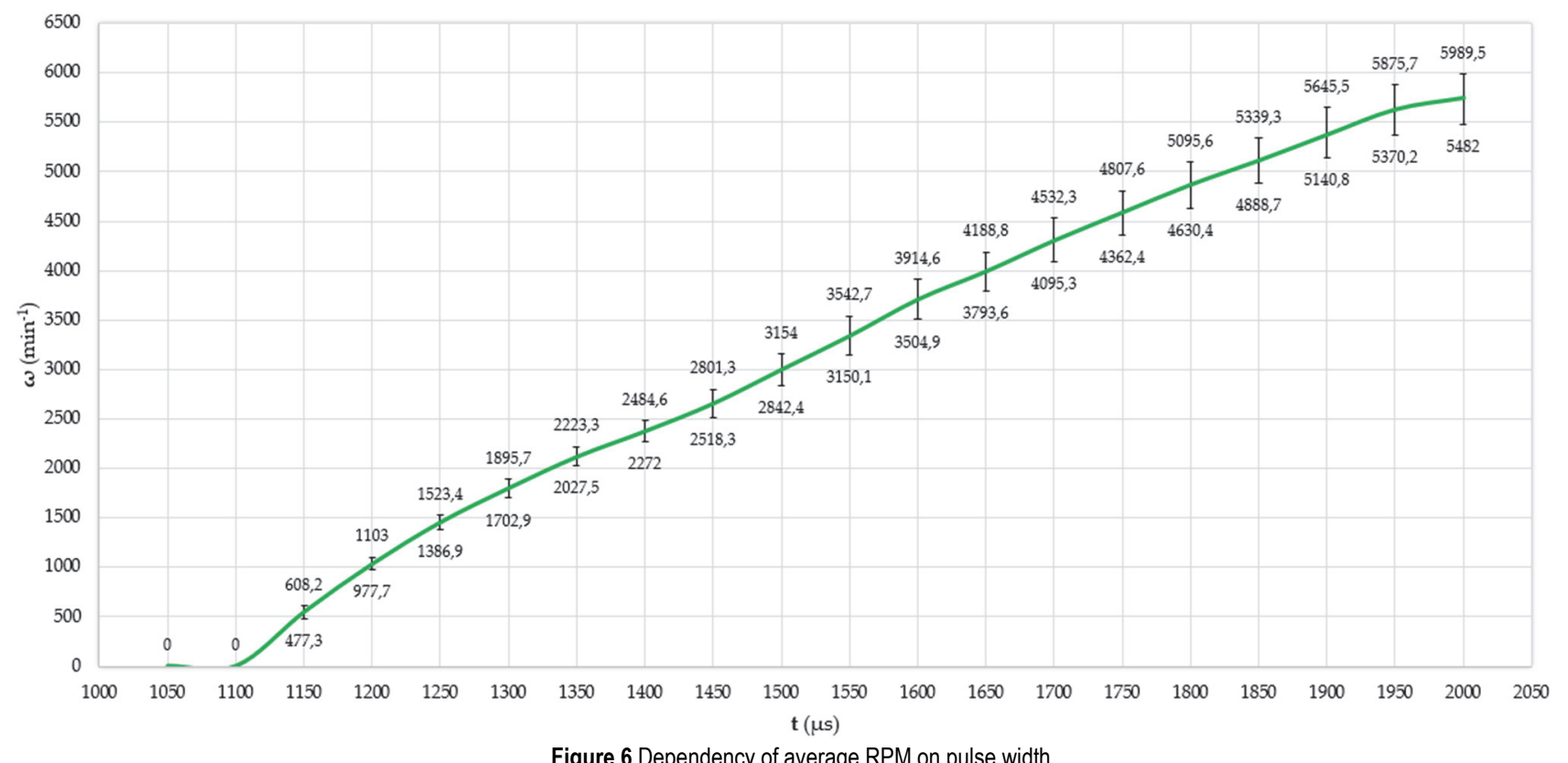

Since the results from measurements at 7 different fixed voltages $(22-25 \mathrm{~V})$ are too close to each other, we decided to present the curve of the average measured RPM and the minimal (value below the curve) and maximal value (value above the curve). The measurements have showed that the maximal average RPM (at $25 \mathrm{~V}$ voltage) of the motor is 5989,5 $\mathrm{min}^{-1}$ and the minimal average RPM (at $22 \mathrm{~V}$ voltage) is $5482 \mathrm{~min}^{-1}$. The dependency also shows that the percentage difference between maximal and minimal value of the RPM is from $1350 \mu$ s pulse width stabilizing at average $9,344 \% \pm 1 \%$ approximately.

Another, very important parameter is the current consumption at different rotational speeds. The dependency of average current consumption on RPM is shown in Fig. 7.

The created dependency shows that the maximal average current consumption of the BLDC motor is 25,86 A (at 5989,5 $\mathrm{min}^{-1}$ ) and the minimal average current consumption is $21,2 \mathrm{~A}$ (at $5482 \mathrm{~min}^{-1}$ ). The dependency also shows that the percentage difference between maximal and minimal value of the current is from the value of RPM measured also at $1350 \mu$ s pulse width stabilizing at average $17,243 \% \pm 2 \%$ approximately. Since the maximal average current is much lower than the ESC's maximal current for this motor-propeller configuration, a cheaper ESC with maximal current of $30 \mathrm{~A}$ can be used.

The last dependency we measured, the dependency of average generated thrust on RPM, is shown in Fig. 8. Since the temperature of the air is between the interval $23^{\circ} \mathrm{C} \pm 5$ ${ }^{\circ} \mathrm{C}$, which is declared by the manufacturer of the sensor to the defined accuracy, the accuracy of the sensor is not affected by the air flow. That means it is not necessary to check the influence of the temperature on the force sensor.

The dependency shows that the maximal generated average thrust at maximal RPM is $32,79 \mathrm{~N}$ and the minimal average thrust at maximal RPM is 28,03 N. After conversion to kilograms, these measurements show that one propeller can lift approximately $3,34 \mathrm{~kg}$ (maximally). 


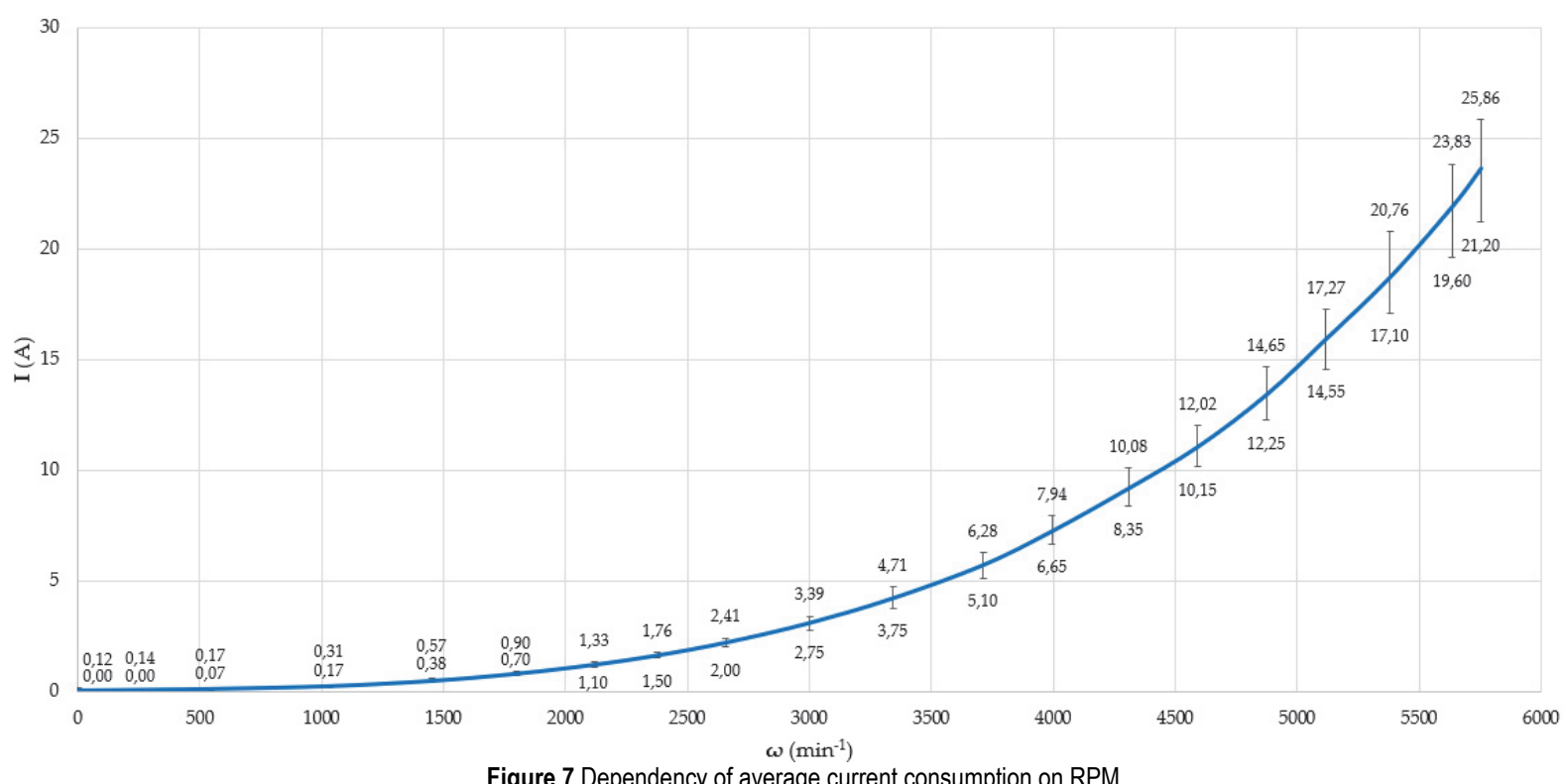

Figure 7 Dependency of average current consumption on RPM

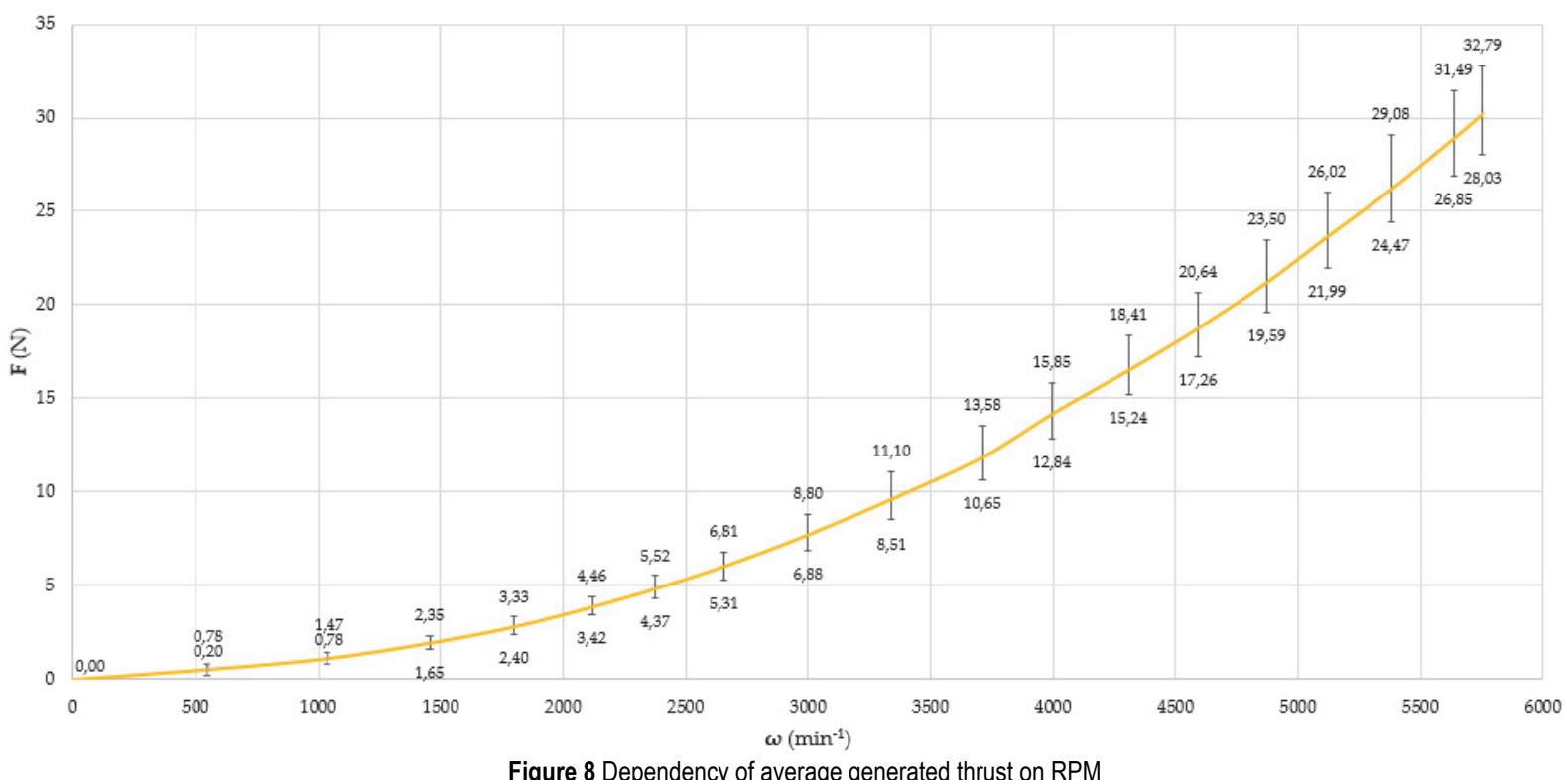

The measurement of force has unlike the previous dependencies shown, that the percentage difference between the maximal and minimal value is exponentially decreasing from the initial value measured at the value of RPM at $1150 \mu$ s pulse width (75\% difference) to $14,512 \%$ difference at the maximal RPM. The variances between the measured values are caused by the difference between supply voltages in each measurement. The change of the supply voltage affects the reachable RPM at every pulse width and the values of each parameter raise slower.

\subsection{Interpretation and Verification of the Measured Data}

To describe the behaviour of the measured propeller by mathematical equations, several regression methods had to be tested. Since the dependencies in Figs. 6 to 8 have polynomial or nonlinear characteristics, our goal was to find a regression model with the tightest possible fit to the original curves.

The first models we analysed were the polynomial regression models. The tightest fit for all dependencies was achieved by the cubic regression model. The regression has showed that all the dependencies can be interpreted with coefficient of determination between 0,998 and 0,999 . The equation for the cubic regression model takes the following form:

$y=a_{\mathrm{c}}+b_{\mathrm{c}} x+c_{\mathrm{c}} x^{2}+d_{\mathrm{c}} x^{3}$

where $a_{\mathrm{c}}, b_{\mathrm{c}}, c_{\mathrm{c}}$ and $d_{\mathrm{c}}$ are the coefficients for each dependency. The values of these coefficients are shown in Tab. 2.

Table 2 Coefficient values for cubic regression model

\begin{tabular}{|c|c|c|c|c|}
\hline Dependency & $R^{2}$ & Coefficient & Value & Standard error \\
\hline \multirow{4}{*}{$\begin{array}{l}\text { RPM on pulse } \\
\text { width }\end{array}$} & \multirow{4}{*}{0,998} & $a_{\mathrm{c}}$ & $-8194,45$ & $\pm 3648,087$ \\
\hline & & $b_{\mathrm{c}}$ & 6,974 & $\pm 7,442$ \\
\hline & & $c_{\mathrm{c}}$ & 0,001 & $\pm 0,005$ \\
\hline & & $d_{\mathrm{c}}$ & $-6,818 \cdot 10^{-7}$ & $\pm 1,083 \times 10^{-6}$ \\
\hline \multirow{4}{*}{$\begin{array}{l}\text { Current on } \\
\text { RPM }\end{array}$} & \multirow{4}{*}{0,999} & $a_{\mathrm{c}}$ & 0,042 & $\pm 0,085$ \\
\hline & & $b_{\mathrm{c}}$ & $6,654 \times 10^{-4}$ & $\pm 1,347 \times 10^{-4}$ \\
\hline & & $c_{\mathrm{c}}$ & $-4,124 \times 10^{-7}$ & $\pm 5,717 \times 10^{-5}$ \\
\hline & & $d_{\mathrm{c}}$ & $1,747 \times 10^{-10}$ & $\pm 6,589 \times 10^{-12}$ \\
\hline \multirow{4}{*}{$\begin{array}{c}\text { Thrust on } \\
\text { RPM }\end{array}$} & \multirow{4}{*}{0,999} & $a_{\mathrm{c}}$ & 0,07 & $\pm 0,06$ \\
\hline & & $b_{\mathrm{c}}$ & $1,459 \times 10^{-4}$ & $\pm 9,505 \times 10^{-5}$ \\
\hline & & $c_{\mathrm{c}}$ & $7,249 \times 10^{-7}$ & $\pm 4,035 \times 10^{-8}$ \\
\hline & & $d_{\mathrm{c}}$ & $2,812 \times 10^{-11}$ & $\pm 6,651 \times 10^{-12}$ \\
\hline
\end{tabular}


The other regression model we tested was the exponential regression. Best results were achieved by the following equation:

$$
y=a_{\mathrm{e}}+b_{\mathrm{e}} \mathrm{e}^{c_{\mathrm{e}} x}
$$

where $a_{\mathrm{e}}, b_{\mathrm{e}}, c_{\mathrm{e}}$ are the coefficients for each dependency. The values of these coefficients are shown in Tab. 3 .

Table 3 Coefficient values for exponential regression model

\begin{tabular}{|c|c|c|c|c|}
\hline Dependency & $R^{2}$ & Coefficient & Value & Standard error \\
\hline \multirow{2}{*}{$\begin{array}{c}\text { RPM on } \\
\text { pulse width }\end{array}$} & \multirow{2}{*}{0,998} & $a_{\mathrm{e}}$ & 14745,088 & $\pm 1833,565$ \\
\cline { 3 - 5 } & & $b_{\mathrm{e}}$ & $-26436,088$ & $\pm 838,088$ \\
\cline { 3 - 5 } & $c_{\mathrm{e}}$ & $-5,448$ & $\pm 8,589 \times 10^{-5}$ \\
\hline \multirow{2}{*}{$\begin{array}{c}\text { Current on } \\
\text { RPM }\end{array}$} & \multirow{2}{*}{0,999} & $a_{\mathrm{e}}$ & $-0,986$ & $\pm 0,495$ \\
\cline { 3 - 5 } & & $b_{\mathrm{e}}$ & 0,639 & $\pm 3,867 \times 10^{-7}$ \\
\cline { 3 - 5 } & $c_{\mathrm{e}}$ & $6,364 \times 10^{-4}$ & $\pm 1,342 \times 10^{-5}$ \\
\hline \multirow{2}{*}{$\begin{array}{c}\text { Thrust on } \\
\text { RPM }\end{array}$} & \multirow{2}{*}{0,998} & $a_{\mathrm{e}}$ & $-4,889$ & $\pm 0,608$ \\
\cline { 3 - 5 } & & $b_{\mathrm{e}}$ & $-4,238$ & $\pm 0,415$ \\
\cline { 3 - 5 } & $c_{\mathrm{e}}$ & $3,702 \times 10^{-4}$ & $\pm 1,498 \times 10^{-5}$ \\
\hline
\end{tabular}

The last model we analysed was the proportional rate regression. Best results were achieved by the following equation:

$$
y=a_{\mathrm{p}}-\frac{b_{\mathrm{p}}}{c_{\mathrm{p}}}\left(1-\mathrm{e}^{-c_{\mathrm{p}} x}\right)
$$

where $a_{\mathrm{p}}, b_{\mathrm{p}}$ and $c_{\mathrm{p}}$ are the coefficients for each dependency. The values of these coefficients are shown in Tab. 4.

Table 4 Coefficient values for proportional rate regression model

\begin{tabular}{|c|c|c|c|c|}
\hline Dependency & $R^{2}$ & Coefficient & Value & Standard error \\
\hline \multirow{2}{*}{$\begin{array}{c}\text { RPM on } \\
\text { pulse width }\end{array}$} & \multirow{2}{*}{0,998} & $a_{\mathrm{p}}$ & -11691 & \pm 1055 \\
\cline { 3 - 5 } & & $b_{\mathrm{p}}$ & $-14,403$ & $\pm 1,849$ \\
\cline { 3 - 5 } & $c_{\mathrm{p}}$ & $54,483 \times 10^{-5}$ & $\pm 8,589 \times 10^{-5}$ \\
\hline \multirow{2}{*}{$\begin{array}{c}\text { Current on } \\
\text { RPM }\end{array}$} & \multirow{2}{*}{0,999} & $a_{\mathrm{p}}$ & $-0,347$ & $\pm 0,11$ \\
\cline { 3 - 5 } & & $b_{\mathrm{p}}$ & $-40,667 \times 10^{-5}$ & $\pm 2,334 \times 10^{-5}$ \\
\hline & $c_{\mathrm{p}}$ & $-63,638 \times 10^{-5}$ & $\pm 1,342 \times 10^{-5}$ \\
\hline \multirow{2}{*}{$\begin{array}{c}\text { Thrust on } \\
\text { RPM }\end{array}$} & \multirow{2}{*}{0,998} & $a_{\mathrm{p}}$ & $-6,651$ & $\pm 0,229$ \\
\cline { 3 - 5 } & & $b_{\mathrm{p}}$ & $-1,569 \times 10^{-3}$ & $\pm 9,07 \times 10^{-5}$ \\
\cline { 3 - 5 } & $c_{\mathrm{p}}$ & $37,021 \times 10^{-5}$ & $\pm 1,498 \times 10^{-5}$ \\
\hline
\end{tabular}

According to the tested regression models, the tightest fit in all dependencies has the cubic regression.

With this regression model of the propeller's parameters it is possible to calculate its behaviour when the measurements can be out of the limit of the used BLDC motor's RPM or torque (e.g. measurement with a different BLDC motor with higher maximal RPM).

\section{DISCUSSION}

According to the cited approaches, where the researchers are using only single purpose systems [1-3], where their solution can measure only a few motorpropeller combinations and where in some cases measurement of several quantities (e. g. current consumption, RPM etc.) is missing [4] or they use inaccurate measurement devices or sensors (e. g. panel voltmeter/ammeter on the power supply) $[1-3,5,7]$, our solution provides an innovative and compact method for measurement of several unknown parameters of a wide range of motor/propeller combinations by calibrated sensors with defined accuracy. In comparison with methods cited in [1-3] our system is not directly integrated to a microcontroller, so it is possible to use it with several kinds of embedded boards (Raspberry Pi, STM32 etc.).

A very common issue was also the missing method for storage of measured values, where the measured data was written down only by an operator that controlled the measurement cycle [1-3] or it was sent by Bluetooth or USB to the computer for further analysis [4, 5, 7]. Our proposed solution is based on an automatic measurement cycle, where 10 measurements of each quantity are performed to obtain the best possible data. After the measurement, the data is saved to a text file and prepared for the next processing. This way of data management allows raising the repeatability of the measurement by knowing the value of the data at each time step.

Other novel approach in comparison to the cited papers [1-9] is the fact that we have designed a measurement system that is compatible with most of the used BLDC motors on medium and large sized UAVs.

When we compare the results of the regression analysis with the interpretation described by Szafranski [8] and Ryll [9] we found out, that their dependencies on different propellers were similarly approximated by cubic functions like it was in our case. Other authors in [1-7] had very similar dependencies, but unfortunately, they did not publish a regression model that would describe the acquired data.

Furthermore, it would be interesting to investigate more similar propellers and their behaviour in comparison with the one measured in this article. Measurements with different propellers can give us more information about their parameters and about their mathematical description.

\section{CONCLUSIONS}

In this paper, a design of an experimental stand for measurement of a UAV propeller's thrust is presented. The presented custom device is applicable for measurement of several types of BLDC motors and propellers with the possibility to measure BLDC motors with current consumption up to $100 \mathrm{~A}$. The novelty of the system is mainly based on development of a custom measurement board that will acquire data from the sensors, process it and save it for future use. On the other hand, this board can be used with any kind of embedded boards that has the needed peripherals. Our experiments with a Tarot $5008340 \mathrm{KV}$ BLDC motor and a Dragonfly 1755 propeller with unknown parameters have helped to understand its behaviour in practice. On the other hand, the measured dependencies have shown that the relation between the parameters can be described by a cubic regression model with different coefficients. This way of the description of the propeller's parameters can be used as a method to calculate its parameters in unmeasurable conditions (e. g. higher RPM than the motor's maximal).

The measured data also shows that for this motorpropeller configuration it is enough to use an ESC with lower maximal current (e. g. 30 A). The results of the measurements also contribute to detailed estimation of the behaviour of the motor-propeller pair in conditions when the battery is discharging. This kind of description allows in practice to rise the flight time of a UAV on which the 
propellers will be placed. The other contribution of the done measurements is that the measured data at different voltages makes it possible to predict flight failures that can be caused by the loss of the propellers thrust when a payload is transported.

Further work will include a research of the propeller's behaviour in different environment (effect of temperature and humidity on the parameters). Since the designed measurement stand is a prototype, the placement and the way of RPM measurement will be improved and the effect of the motor's position on measured values will be analysed. To reduce the effect of the designed hardware components on the air flow near the propeller further study and fluid flow simulation will be done to optimize the solution and to reduce the ceiling effect.

\section{Acknowledgements}

This paper was created within the project VEGA No. 1/0720/18 Research of Alternative Navigation Algorithms for the Control of Autonomous Robots in Plant Production.

\section{REFERENCES}

[1] Jakubowski, A., Kubacki, A., Minorowicz, B., \& Nowak, A. (2015). Analysis Thrust for Different Kind of Propellers. Advances in Intelligent Systems and Computing Progress in Automation, Robotics and Measuring Techniques, 85-90. https://doi.org/10.1007/978-3-319-15796-2_9

[2] Zabunov, S. \& Mardirossian, G. (2018). Scales for measuring UAV micro-motor static thrust. Aerospace Research in Bulgaria, 30, 96-102. https://doi.org/10.3897/arb.v30.e08

[3] Theys, B., Dimitriadis, G., Hendrick, P., \& Schutter, J. D. (2016). Influence of propeller configuration on propulsion system efficiency of multi-rotor Unmanned Aerial Vehicles. 2016 International Conference on Unmanned Aircraft Systems (ICUAS). https://doi.org/10.1109/icuas.2016.7502520

[4] Hossain, M. R. \& Krouglicof, N. (2010). Propeller dynamometer for small Unmanned Aerial Vehicle. Ccece 2010. https://doi.org/10.1109/ccece.2010.5575152

[5] Virginio, R., Fuad, F. A., Jihadil, M., Ramadhani, M. J., Rafie, M., Stevenson, R., \& Adiprawita, W. (2018). Design and implementation of low cost thrust benchmarking system (TBS) in application for small scale electric UAV propeller characterization. Journal of Physics: Conference Series, 1130. https://doi.org/10.1088/1742-6596/1130/1/012022

[6] Muzar, D. \& Lanteigne, E. (2016). Experimental characterization of brushless dc motors and propellers for flight application. Proceedings of the Canadian Society for Mechanical Engineering International Congress 2016.

[7] Kotarski, D., Krznar, M., Piljek, P., \& Simunic, N. (2017). Experimental Identification and Characterization of Multirotor UAV Propulsion. Journal of Physics: Conference Series, 870, 012003. https://doi.org/10.1088/1742-6596/870/1/012003

[8] Szafranski, G., Czyba, R., \& Blachuta, M. (2014). Modeling and identification of electric propulsion system for multirotor unmanned aerial vehicle design. 2014 International Conference on Unmanned Aircraft Systems (ICUAS). https://doi.org/10.1109/icuas.2014.6842287

[9] Ryll, M., Bulthoff, H. H., \& Giordano, P. R. (2015). A Novel Overactuated Quadrotor Unmanned Aerial Vehicle: Modeling, Control, and Experimental Validation. IEEE Transactions on Control Systems Technology, 23(2), 540556. https://doi.org/10.1109/tcst.2014.2330999
[10] Conyers, S. A. (2019). Empirical Evaluation of Ground, Ceiling, and Wall Effect for Small-Scale Rotorcraft. Retrieved from: https://digitalcommons.du.edu/etd/1570

[11] Danjun, L., Yan, Z., Zongying, S., \& Geng, L. (2015). Autonomous landing of quadrotor based on ground effect modelling. 2015 34th Chinese Control Conference (CCC). https://doi.org/10.1109/chicc.2015.7260521

[12] Cheeseman, I. C. \& Bennett, W. E. (1957). The effect of the ground on a helicopter rotor in forward flight. Technical report, Aeronautical Research Council.

[13] Wei, P., Chan, S. N., Lee, S., \& Kong, Z. (2019). Mitigating ground effect on mini quadcopters with model reference adaptive control. International Journal of Intelligent Robotics and Applications, 3(3), 283-297. https://doi.org/10.1007/s41315-019-00098-z

[14] Sanchez-Cuevas, P. J., Heredia, G., \& Ollero, A. (2017). Multirotor UAS for bridge inspection by contact using the ceiling effect. 2017 International Conference on Unmanned Aircraft Systems (ICUAS). https://doi.org/10.1109/icuas.2017.7991412

[15] Kocer, B. B., Tjahjowidodo, T., \& Seet, G. G. (2018). Centralized predictive ceiling interaction control of quadrotor VTOL UAV. Aerospace Science and Technology, 76, 455-465. https://doi.org/10.1016/j.ast.2018.02.020

\section{Contact information:}

Patrik KósA, PhD, M. Eng.

(Corresponding author)

Information and Coordination Centre of Research, Faculty of Engineering,

Slovak University of Agriculture in Nitra, Tr. A. Hlinku 2, 94976 Nitra, Slovakia

E-mail: patrik.kosa@uniag.sk

\section{Marián KIŠEV, M. Eng.}

Department of Electrical Engineering, Automation and Informatics,

Faculty of Engineering, Slovak University of Agriculture in Nitra,

Tr. A. Hlinku 2, 94976 Nitra, Slovakia

E-mail: xkisevm@is.uniag.sk

\section{Lukáš VACHO, PhD, M. Eng.}

Department of Electrical Engineering, Automation and Informatics,

Faculty of Engineering, Slovak University of Agriculture in Nitra,

Tr. A. Hlinku 2, 94976 Nitra, Slovakia

E-mail: lukas.vacho@uniag.sk

Ladislav TÓTH, PhD, M. Eng.

Department of Electrical Engineering, Automation and Informatics, Faculty of Engineering, Slovak University of Agriculture in Nitra,

Tr. A. Hlinku 2, 94976 Nitra, Slovakia

E-mail: ladislav.toth@uniag.sk

Martin OLEJÁR, Associate Professor, PhD, M. Eng.

Department of Electrical Engineering, Automation and Informatics,

Faculty of Engineering, Slovak University of Agriculture in Nitra,

Tr. A. Hlinku 2, 94976 Nitra, Slovakia

E-mail: martin.olejar@uniag.sk

Marta HARNIČÁROVÁ, PhD, M. Eng.

Department of Electrical Engineering, Automation and Informatics,

Faculty of Engineering, Slovak University of Agriculture in Nitra,

Tr. A. Hlinku 2, 94976 Nitra, Slovakia

E-mail: marta.harnicarova@uniag.sk

Jan VALÍČEK, Associate Professor, PhD, M. Eng.

Department of Electrical Engineering, Automation and Informatics, Faculty of Engineering, Slovak University of Agriculture in Nitra,

Tr. A. Hlinku 2, 94976 Nitra, Slovakia

E-mail: jan.valicek@uniag.sk

Hakan TOZAN, Associate Professor, PhD

School of Engineering and Natural Sciences, Istanbul Medipol University,

Istanbul, 34810, Turkey

E-mail: htozan@medipol.edu 